\title{
Evolución a largo plazo de la fluxión funículo testicular en la reparación herniaria inguinal*
}

\author{
Drs. ALBERTO ACEVEDO F. ${ }^{1}$, JORGE BRAVO L. ${ }^{1}$, VERÓNICA DELLEPIANE T. ${ }^{1}$ \\ 1 Centro de Referencia de Salud (CRS) Cordillera Oriente, Unidad de Cirugía Mayor Ambulatoria. \\ Universidad de Chile, Facultad de Medicina, Campus Peñalolén. \\ Santiago, Chile.
}

\section{Funiculus testicular complications in inguinal hernia repair. Long term evolution}

\section{Introducción}

En un estudio previo ${ }^{1}$, comprobamos que el aumento de volumen del funículo espermático y del testículo fue la complicación más frecuentemente observada en los pacientes intervenidos de una hernia inguinal. Destacamos la escasa mención que de ella se hace en la literatura especializada y el desconocimiento existente respecto a su frecuencia y evolución. Con el fin de precisar la extensión del término denominamos fluxión funiculotesticular (FFT) al aumento de volumen, indoloro o escasamente doloroso, que no produce fiebre, compromiso del estado general ni incapacidad funcional, diferenciándose del cuadro de la orquitis isquémica, en que ésta, junto con la fluxión se acompaña de dolor, incapacidad funcional, fiebre y compromiso del estado general. La atrofia testicular es la evolución en el tiempo de la orquitis isquémica y se caracteriza por un testículo pequeño, aumentado de consistencia e indoloro, la evolución en el tiempo de la FFT ha sido poco estudiada.

Como señaláramos con anterioridad ${ }^{1}$, el cordón espermático tiene un diámetro inferior a $5 \mathrm{~mm}$ en los sujetos sanos. Se clasificó su aumento de grosor como leve (0,5-1 cm), moderado $(1-2 \mathrm{~cm})$, y severo $(>2 \mathrm{~cm})$. El aumento de volumen y de consistencia del testículo es de muy difícil objetivación y por ello nos limitamos a señalar su presencia. En esta presentación damos cuenta de la evolución en el tiempo de la FFT en los 67 pacientes que desarrollaron una FFT consecutiva a una herniorrafia inguinal.

El estudio de los pacientes se realizó tras un seguimiento entre 3 y 5 años. El control fue efectuado personalmente por un miembro del equipo, en la totalidad de los enfermos los que fueron citados al CRS con este propósito. Se examinó palpatoriamente el grosor del funículo y el volumen testicular buscando específicamente una atrofia testicular. Esta información se vertió en la base de datos del Centro.

\section{$\underline{\text { Resultados del seguimiento }}$}

En la totalidad de los 67 pacientes con FFT en todos sus grados se apreció una regresión lenta de los síntomas. El control efectuado en los pacientes con fluxiones leves o moderadas demostró una regresión del engrosamiento funicular a valores normales $(<0,5 \mathrm{~cm})$ en todos los casos. No se detectó en ellos atrofia testicular.

De los 8 pacientes con fluxión severa, 5 habían recuperado el grosor normal del deferente y el tamaño del testículo y 3 presentaban un engrosamiento residual, leve $(<1 \mathrm{~cm})$ del cordón espermático. En estos 3 pacientes se apreció un aumento del volumen

*Recibido el 12 de marzo de 2010 y aceptado para publicación el 11 de enero de 2011.

Correspondencia: Dr. Alberto Acevedo F.

Las Torres 5100, Santiago, Chile. aacevedof@crsoriente.cl 
del testículo con relación al contra lateral, pero conservando su consistencia normal. La ecotomografía reveló normalidad de la trama tisular en todos ellos y un leve hidrocele en dos casos.

Dentro de la evolución, por lo general favorable, de la cirugía herniaria, no deja de ser preocupante para el enfermo y para el médico la comprobación del aumento de volumen y consistencia del cordón espermático y del testículo en el postoperatorio inmediato, especialmente en su forma severa de esta complicación ${ }^{2-6}$.

En una publicación anterior señalábamos que la FFT se aprecia con frecuencia en la herniorrafia inguinal y que afecta con mayor frecuencia a aquellos pacientes que requieren de una mayor disección durante la cirugía, es decir, a las hernias recidivadas, a las de gran tamaño y a las inguinoescrotales ${ }^{1,7-9}$.

Ya en 1984, Wanz ${ }^{10}$, había señalado el compromiso circulatorio responsable de la "orquitis isquémica” y había sugerido que su génesis podía ser arterial o venosa. Especial preponderancia a este respecto tuvieron las observaciones de Fruchaud ${ }^{11}$, quien demostró en numerosos pacientes que se trataba de una isquemia por compromiso de la circulación venosa y trombosis del plexo pampiniforme.

Analizando pragmáticamente este cuadro Ponka ${ }^{12}$, señala que si bien, excepcionalmente, la orquitis isquémica puede deberse al daño de la circulación arterial, el mismo cuadro clínico podrían ser la consecuencia de una trombosis venosa. Él describe que en ciertas raras oportunidades el cordón aparece engrosado y el testículo aumentado de tamaño asociado a dolor y alza febril. Consciente de la dificultad de establecer su etiología, Ponka prefiere hablar de swelling due to venous obstruction (fluxión por obstrucción venosa).

Nosotros coincidimos con Ponka en que la fluxión del funículo y del testículo es lo que mejor define los hallazgos postoperatorios a los que nos estamos refiriendo, concordando que la patogenia es compleja, y que el trauma quirúrgico juega un rol importante en su génesis.

La FFT debe diferenciarse de una "orquitis isquémica”. En ésta el aumento de volumen funicular y testicular será doloroso, impidiendo al paciente reiniciar sus actividades habituales y se acompañará de compromiso del estado general y elevación moderada de la temperatura ${ }^{7-12}$. La FFT, en cambio es indolora o escasamente dolorosa, no impide al paciente realizar sus actividades habituales y no se acompaña de alza febril.

La FFT pone en evidencia un manejo inadecuado de los tejidos ${ }^{13,14}$. La etapa inflamatoria será más prolongada e intensa si a la cadena de hechos propios de la cicatrización se suman los mecanismos necesarios para resolver una interfase cicatricial alterada por tejidos degradados o desprovistos de circulación y hematomas. Todas las maniobras destinadas a disminuir el trauma quirúrgico contribuirán a prevenir una FFT.

El trauma quirúrgico puede inducir, por si mismo, una trombosis venosa del plexo pampiniforme la que, en casos graves, interferirá con la circulación del testículo y desencadenará el grave cuadro de la isquemia testicular ${ }^{11,13-19}$.

Tuvimos la oportunidad de observar la evolución de una orquitis isquémica en un paciente intervenido en otro Centro. Efectuamos controles periódicos de la circulación de los vasos testiculares con la técnica de duplex comprobando una progresiva disminución del flujo pulsátil de la arteria testicular que se acentuó hasta el cese completo del flujo. Doce semanas después de la intervención, en el control alejado, la inflamación y el edema habían regresado, poniendo en evidencia la atrofia del testículo afectado.

Los aspectos médico legales de esta complicación deben ser convenientemente sopesados. Parece recomendable a este respecto que las hernias de gran tamaño, inguinoescrotales y recidivadas sean intervenidas por cirujanos con experiencia en cirugía herniaria y que el riesgo sea comunicado al paciente al momento de obtener el consentimiento informado. Gilbert $^{20}$, señala que el edema testicular hace infeliz al paciente y ello es aun peor si evoluciona hacia una isquemia y atrofia testicular. Señala que cualquier cirujano está expuesto a esta complicación y establece que una relación próxima entre el médico y el paciente es la mejor manera de prevenir graves consecuencias; la protocolización del estudio preoperatorio dejando constancia específicamente de los hallazgos preoperatorios en el funículo y el testículo, y el registro de los hallazgos durante el acto quirúrgico son elementos imprescindibles al momento de prevenir graves consecuencias médicolegales.

De estas observaciones es posible concluir que la fluxión funículo testicular se diferencia en forma neta de la isquemia testicular y tiene una evolución benigna aún en las formas más graves.

\section{Referencias}

1. Acevedo A, Gallego A, Viterbo A, Bravo J, Dellepiane V. Fluxión funículo-testicular, una complicación frecuente de la herniorrafia inguinal. Rev Chil Cir. 2007; 59: 55-60.

2. Bendavid R. Complications of groin hernia surgery. Surg Clin North Am. 1998;78: 1089-101.

3. Pollak R, Nyhus LM. Complications of groin hernia repair. Surg Clin North Am. 1983;63:11363-71.

4. Wantz GE. Testicular complications of inguinal hernioplasty. Probl Gen Surg. 1995;12:219-24. 
5. Schumpelick V. Treutner KH, Arlt G. Inguinal hernia repair in adults. Lancet 1994;344:375-9.

6. Kark AE, Kurzer M, Waters KJ. Tension free mesh repair: review of 1098 cases using local anesthesia in a day unit. Ann R Coll Surg Engl. 1995;77:299-304.

7. Bardavid C. Hernias inguinales, Técnica de Shouldice. Rev Chil Cir. 1993;45:264-71.

8. Greco D. Complications in open hernia surgery. En Schumpelick V, Wantz GE. Inguinal hernia repair. Basel, Karger, 1995 pp 320-5.

9. Rötzscher VM, Pleye JT. Hernioplastik nach Schouldice. Qualität und Qualifikation. Aktuel Chir. 1990;25: 263-8.

10. Wantz GE. Complications of inguinal hernial repair. Surg Clin North Am. 1984;64:287-95.

11. Fruchaud H. Le traitement chirugical des hernies de l'aine. Paris, Doin, 1956 pp 195-200.

12. Ponka JL. Hernias of the abdominal wall. WB Sounder Co, Philadelphia. 1980 pp 608-9.

13. Levy AH, Wreen R, Friedman MN. Complications and recurrences after inguinal hernia repair. Ann Surg
1951;133:533-9.

14. Madden JL, Hakim S, Agorogiannis AB. The anatomy and repair of inguinal hernias. Surg Clin North Am. 1971;51:1269-92.

15. KurzerM, Kark A, Wantz G. Surgical management of abdominal wall hernias. Martin Dunitz Ltd, London, 1999 pp 108-93.

16. Klinge U. Complications in open surgery. En Schumpelick V Wantz GE. Inguinal hernia repair. Basel, Karger, 1995 pp 326-39.

17. Richards AT. Cord and testicular complications of groin hernia surgery, en Fitzgibbons R J, Greenburg A G. Nyhus and Condon's hernia. Lippincott Williams \& Williams, Philadelphia, 2002 pp 291-6.

18. Wanz GE. Perfusion of the testicles and ischemic orchitis. En Schumpelick V, Wantz GE. Inguinal hernia repair. Basel, Karger, 1995 pp 345-53.

19. Gilbert AI. Medical legal aspects of hernia surgery: Personal risk management. En Schumpelick V, Wantz GE. Inguinal Hernia Repair. Expert meeting on hernia surgery, 1994, Basel, Karger, 1995 pp 416-28. 